\title{
Transmission IR Micro-Spectroscopy of Interfacial Water between Colloidal Silica Particles*
}

\author{
Mai Hamamoto, Makoto Katsura, Naoki Nishiyama, Ryota Tononue, and Satoru Nakashima ${ }^{\dagger}$ \\ Department of Earth and Space Science, Graduate School of Science, \\ Osaka University, Machikaneyama-cho 1-1, Toyonaka, Osaka 560-0043, Japan
}

(Received 31 December 2014; Accepted 13 May 2015; Published 20 June 2015)

\begin{abstract}
Interfacial water among silica particles were measured on silica colloid suspensions (0-31.1 vol.\%) by transmission infrared micro-spectroscopy. The difference absorption index $k$ spectra from the pure water of silica colloid suspensions with varying volume fractions have residual components in the $\mathrm{O}-\mathrm{H}$ stretching region: the $3060 \mathrm{~cm}^{-1}$ component increased linearly with the silica volume fraction, while the $3620 \mathrm{~cm}^{-1}$ component increased and the $3380 \mathrm{~cm}^{-1}$ component decreased with much less linearity. The 3060 and $3620 \mathrm{~cm}^{-1}$ components are considered to be characteristic to the surface silanol and interfacial water and affected by overlapping diffuse electrical double layers among silica particles.

[DOI: $10.1380 /$ ejssnt.2015.301]
\end{abstract}

Keywords: Infrared absorption spectroscopy; Water; Silicon oxides; Nano-particles; Surface structure

\section{INTRODUCTION}

Liquid water at solid surfaces has been reported to have some properties different from those of bulk based on experiments and computer simulations. Such water, called as interfacial water, is thought to play a key role in geological, biological, environmental, and material sciences [13]. The water-mineral interaction between the interfacial water and minerals is thought to be important for understanding the mineral dissolution, fluid flow and weathering processes [4]. Interfacial water spreading in the range of several water molecular layers $(\sim 1-2 \mathrm{~nm})$ has been studied in many experiments such as surface force apparatus (SFA) [5, 6], X-ray scattering [7, 8], atomic force microscopy (AFM) [9], and vibrational spectroscopy.

Concerning the vibrational spectroscopy, infrared (IR) measurements were often used to investigate the water structure, because the $\mathrm{O}-\mathrm{H}$ stretching band reflects hydrogen bond distances [10]. In particular, sum-frequency generation (SFG) and second harmonic generation (SHG) spectroscopy were often used to measure the interfacial water at solid/water boundaries. SFG and SHG have been used for solid/water or air/water interfaces [11-19], because of their selectivity of the interfacial water due to its molecular orientation.

Silica is one of the representative solid earth's surface materials and have also been studied by material scientists. Silica/water interfaces were studied by SFG [15-17] and liquid-like $\left(\sim 3400 \mathrm{~cm}^{-1}\right)$ and ice-like $\left(\sim 3200 \mathrm{~cm}^{-1}\right)$ water components were found on silica surfaces.

Interfacial water in solid/water/solid system, which is called as confined water, has been studied mostly by molecular dynamic simulations [20-23]. Modified ion distributions in pore waters of silica [20] and mica [21] have been observed. The diffusion coefficients were found to be smaller for pore sizes less than about $1 \mathrm{~nm}[21,22]$.

\footnotetext{
* This paper was presented at the 7th International Symposium on Surface Science, Shimane Prefectural Convention Center $(\mathrm{Ku}-$ nibiki Messe), Matsue, Japan, November 2-6, 2014.

$\dagger$ Corresponding author: satoru@ess.sci.osaka-u.ac.jp
}

Anomalous dielectric constants in nanopores $(1.2 \mathrm{~nm})$ of silica were also reported [23]. These modified properties can be related to the electrical double layer (EDL) [24]. Water in porous silica as the hydrophilic substrate was often studied experimentally [25-27]. Although numerical simulations such as molecular dynamics (MD) and ab-initio calculation were carried out for explaining experimental results $[28,29]$, there is few quantitative spectroscopic evaluation relating the above simulation and experimental results. Different natures of interfacial waters from the bulk liquid water were explained only qualitatively by the breaking of hydrogen-bonding network surrounding the silica particles [25].

The SFG method characterizes only the water molecules at the very interface. However, in geological environments, water can be present in thicker films (1$100 \mathrm{~nm}$ ) on minerals such as quartz [30], and little is known on the changes of water structure with thickness.

Attenuated total reflection (ATR) IR spectroscopy can be used to study mineral/water interfaces by means of evanescent waves with thick solution layers placed on mineral films. The measured depth of penetration of evanescent waves in the $\mathrm{OH}$ stretching region is about 500 $200 \mathrm{~nm}$ [31]. This depth is too thick to observe structural modifications of interfacial water. Moreover, $\mathrm{OH}$ band shapes are distorted by effects of variation of penetration depth of evanescent wave with wavenumber and anomalous dispersion due to complex refractive index [31]. Therefore, appropriate corrections are necessary to extract structural information on interfacial water.

In this study, quantitative transmission IR spectra are measured on colloidal silica suspensions with appropriate corrections and the spectroscopic features of interfacial water are extracted. The transmission spectroscopy is the most reliable quantitative method following the LambertBeer's law. The concentrations of colloidal silica suspensions were changed in order to vary thicknesses of interfacial water among silica particles. Increase/decrease of different $\mathrm{OH}$ band components of water with decreasing distances among silica particles are determined. The results are discussed based on overlapping electrical double layers spreading on the silica surfaces. 


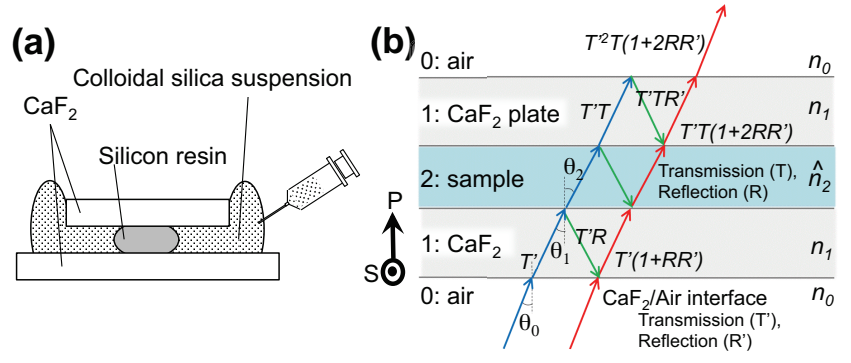

FIG. 1. (a) Schematic figure for the sample setup. Colloidal silica suspension was injected between the top $\mathrm{CaF}_{2}$ plate $\left(6.0 \mathrm{~mm} \varphi, 1.0 \mathrm{~mm}\right.$ thick) and the bottom $\mathrm{CaF}_{2}$ plate (13.0 $\mathrm{mm} \varphi, 1.0 \mathrm{~mm}$ thick) bound by a silicon resin. (b) Incident and refracted lights through a sample sandwiched between two $\mathrm{CaF}_{2}$ plates. Angles $(\theta)$, refractive indices $(n)$ and transmission $(T)$ and reflection $(R)$ at interfaces are indicated.

\section{EXPERIMENTAL}

\section{A. Colloidal silica suspensions}

The colloidal silica suspensions of different concentrations from 0-31.1 vol.\% sandwiched between two $\mathrm{CaF}_{2}$ plates were measured by transmission IR microspectroscopy (Fig. 1a). A commercial colloidal silica suspension was purchased (Ludox LS, Sigma-Aldrich). The original suspension has 16.5 vol.\% of silica particles with a diameter of about $12 \mathrm{~nm}$. It has a density of $1.21 \mathrm{~g} / \mathrm{mL}$ at $25^{\circ} \mathrm{C}$, and surface area of $215 \mathrm{~m}^{2} / \mathrm{g}$ and its $\mathrm{pH}$ is 8.2 . Suspensions with silica concentrations less than 16.5 vol. $\%$ were made by diluting the original suspension with pure water (MilliQ: > $18.2 \mathrm{M} \Omega \cdot \mathrm{cm})$. Suspensions more than 16.5 vol.\% were made by evaporation of the original one by dry air bubbling or heating. According to the manufacturer report, $\mathrm{SO}_{4}^{2-}(\leq 0.02 \mathrm{wt} . \%)$ is included in the original colloidal silica suspension as $\mathrm{Na}_{2} \mathrm{SO}_{4}$, which gives a range of $\sim 10^{-4}-10^{-3} \mathrm{~mol} / \mathrm{L}$ for different concentrations ( 0-31.1 vol.\%).

\section{B. Transmission IR spectroscopy}

Two $\mathrm{CaF}_{2}$ plates of $1 \mathrm{~mm}$ thick were attached together by using silicon grew to fix the gap between the two plates (Fig. 1a). The thickness of this gap was determined to be about $1-2 \mu \mathrm{m}$ by measuring an absorption spectrum of pure water and fitting the spectrum by the absorbance equation shown below (equation (3)) and a reported complex refractive index of pure water [32]. The above suspensions were put between these two $\mathrm{CaF}_{2}$ plates and IR transmission spectra were measured. The small changes of the gap distance $(\leq \sim 2 \%)$ after changing the sample was monitored by the IR absorbance of the silicon grew at $2960 \mathrm{~cm}^{-1}$.

The IR transmission measurements were conducted by using an FTIR micro-spectrometer (FTIR620+IRT30: Jasco Corp.) equipped with a ceramic IR source, a Ge coated $\mathrm{KBr}$ beam splitter and an MCT ( $\mathrm{HgCdTe})$ detector. Cassegrainian focusing and objective mirrors were used in the IR microscope. All the transmission spectra were obtained by collecting 64 scans with a spectral range of $700-7000 \mathrm{~cm}^{-1}, 4 \mathrm{~cm}^{-1}$ wavenumber resolution and $100 \times 100 \mu \mathrm{m}^{2}$ aperture. The background spectrum was first measured through a $2 \mathrm{~mm}$ thick $\mathrm{CaF}_{2}$ plate having the same total thickness of two $\mathrm{CaF}_{2}$ plates. The measured transmission spectra of sample solutions were then recorded as absorbance: $A b s_{\text {meas. }}=-\log _{10}\left(I / I_{0}\right)$ (where $I_{0}$ is the background spectrum). The maximum error range in the $\mathrm{O}-\mathrm{H}$ stretching band region (2600$3800 \mathrm{~cm}^{-1}$ ) of the obtained spectra was estimated as \pm 0.001 in the absorbance unit (dimensionless).

\section{Extraction of absorption index $k$ spectra}

The obtained spectra include effects of reflections at plate/sample and plate/air interfaces (Fig. 1b). These effects cause the distortion of $\mathrm{O}-\mathrm{H}$ stretching band in apparent absorbance spectra. In order to obtain intrinsic absorption features of water molecules, the absorption index $k(\tilde{v})$ is extracted from the measured spectra composed of complex refractive index $(\hat{n}=n+i k)$ with the real $(n(\tilde{\nu}))$ and imaginary $(k(\tilde{\nu}))$ parts. The procedure is mainly after Keefe et al. [33], using Fresnel equations for reflection and is presented below.

Complex refractive index $\hat{n}(\tilde{\nu})$ of the sample solutions should be first determined. The imaginary part, or absorption index $k(\tilde{\nu})$ is calculated by the following equation using the measured absorption spectrum $A b s_{\text {meas. }}$.

$$
k(\tilde{\nu})=\frac{\ln 10 \cdot A b s_{\text {meas. }}(\tilde{\nu})}{4 \pi \tilde{\nu} d}
$$

where $\tilde{\nu}$ is the wavenumber $\left[\mathrm{cm}^{-1}\right]$ and $d$ is the sample thickness $[\mathrm{cm}] . \quad \ln 10$ is the conversion factor from the measured absorbance in common logarithmic scale to the natural logarithmic scale.

The real part $n(\tilde{v})$ of the refractive index at a given wavenumber $\tilde{\nu}_{a}$ is calculated from $k(\tilde{\nu})$ by the following Kramers-Kronig transformation [34, 35].

$$
n\left(\tilde{\nu}_{a}\right)=n_{\infty}+\frac{2}{\pi} P \sum_{i=0}^{\infty} \frac{\tilde{\nu}_{i} k(\tilde{\nu})}{\tilde{\nu}_{i}^{2}-\tilde{\nu}_{a}^{2}} .
$$

$n_{\infty}$ is taken as $n$ values at $590 \mathrm{~nm}$ without absorption for each sample suspension. $P$ is the Cauchy principal value. These values were determined by the following equation [36]:

$$
n=m_{1}+\left(m_{2}-m_{1}\right) v
$$

where $m_{1}$ is the refractive index of the medium (water: 1.333) [37], $m_{2}$ is the refractive index of a silica particle (fused silica: 1.458) [38], and $v$ is the volume fraction of colloidal particles. The validity of this equation was confirmed by the measurements of sample solutions by an Abbé refractometer (TGK, ER-2S).

These obtained sample refractive index $\hat{n}(\tilde{\nu})$ are used to calculate the apparent absorbance. The calculated apparent spectrum can be described by the following equation including reflections at different interfaces (Fig. 1b): 


$$
\operatorname{Abs}_{\text {cal. }}(\tilde{\nu})=-\log \left[\frac{\cos ^{4}\left(\theta_{0}-\theta_{1}\right) T_{S}(\tilde{\nu})\left(1+2 R_{S}(\tilde{\nu}) R_{S}^{\prime}(\tilde{\nu})+T_{P}(\tilde{\nu})\left(1+2 R_{P}(\tilde{\nu}) R_{P}^{\prime}(\tilde{\nu})\right)\right.}{\cos ^{4}\left(\theta_{0}-\theta_{1}\right)+1}\right] .
$$

The expression of reflection and transmission are developed by the Fresnel amplitude coefficients of the reflected and transmitted radiations:

$$
\begin{aligned}
r_{i, j, s} & =\frac{n_{i} \cos \theta_{i}-n_{j} \cos \theta_{j}}{n_{i} \cos \theta_{i}+n_{j} \cos \theta_{j}} \\
t_{i, j, s} & =\frac{2 n_{i} \cos \theta_{i}}{n_{i} \cos \theta_{i}+n_{j} \cos \theta_{j}} \\
r_{i, j, p} & =\frac{n_{j} \cos \theta_{i}-n_{i} \cos \theta_{j}}{n_{j} \cos \theta_{i}+n_{i} \cos \theta_{j}} \\
t_{i, j, p} & =\frac{2 n_{i} \cos \theta_{i}}{n_{j} \cos \theta_{i}+n_{i} \cos \theta_{j}}
\end{aligned}
$$

where

$$
\begin{aligned}
\cos \theta_{1} & =\sqrt{1-\frac{1}{\hat{n}_{1}^{2}} \sin \theta_{0}} \\
\cos \theta_{2} & =\sqrt{1-\frac{1}{\hat{n}_{2}^{2}} \sin \theta_{0}}
\end{aligned}
$$

The incident beam is assumed to be applied from phase $i$ to $j$, and $\theta_{i}$ is an incident angle and $\theta_{j}$ is a refraction angle. We will call the phase 0 for air, the phase 1 for two $\mathrm{CaF}_{2}$ plates and the phase 2 for sample suspension (Fig. 1b).

Incident angles focused on a sample by Cassegrainian objective mirrors are from $17.5^{\circ}$ to $35.0^{\circ}$ in the present IR micro-spectroscopy. The mean incident angle can be calculated as $27.8^{\circ}$.

The reflection at plate/sample interface $R(1 \rightarrow 2)$, the sample transmission $T(1 \rightarrow 2 \rightarrow 1)$ and the reflection at plate/air interface $R^{\prime}(1 \rightarrow 0)$ (Fig. 1b) can be written as the followings reported by Hawrankek et al. [39], respectively:

$$
\begin{aligned}
R & =\frac{2 r^{2}(1-\cos 2 \delta)}{1+r^{4}-2 r^{2} \cos 2 \delta} \\
T & =\frac{t_{12}^{2} t_{21}^{2}}{1+r^{4}-2 r^{2} \cos 2 \delta} \\
R^{\prime} & =r_{10}^{2}
\end{aligned}
$$

where

$$
\begin{aligned}
\delta & =2 \pi \tilde{v} d n_{2} \cos \theta_{2} \\
r_{12} & =-r_{21}=r
\end{aligned}
$$

The value of refractive index $n_{1}$ for $\mathrm{CaF}_{2}$ at the IR wavenumber range was calculated from the dispersion formula [40].

These equations (4-14) are substituted in the equation (3) and $A b s_{\text {calc. }}$ could be obtained. The $k(\tilde{\nu})$ is replaced by the following relation:

$$
k_{\text {new }}(\tilde{\nu})=k_{\text {old }}(\tilde{\nu}) \times \frac{A b s_{\text {meas. }} .}{A b s_{\text {calc. }}} .
$$

The above procedures are repeated until the root mean square of $\left[A b s_{\text {meas. }}-A b s_{\text {calc. }}\right]$ is less than $10 \times 10^{-5}$.

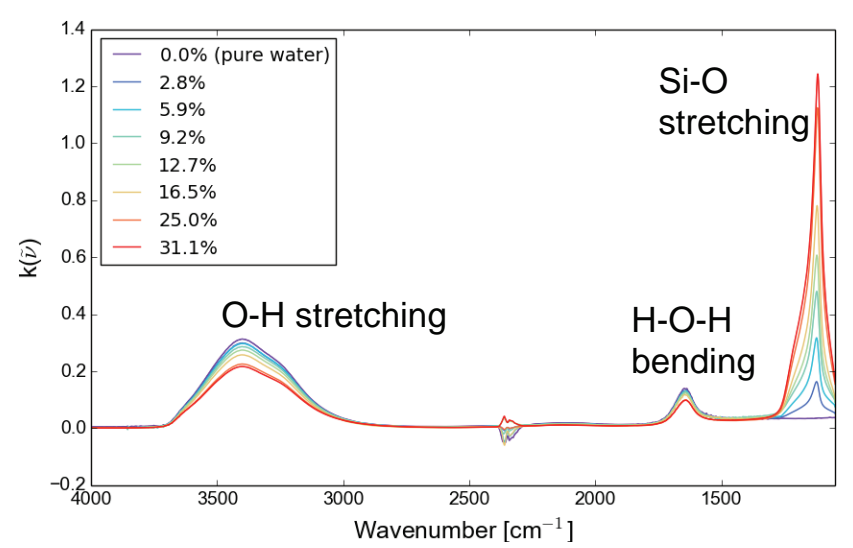

FIG. 2. Absorption index $k$ spectra of the colloidal silica suspensions for different volume fractions (0-31.1 vol.\%).

\section{RESULTS}

\section{A. Absorption index $k$ spectra for colloidal silica suspensions}

The absorption index spectra (Fig. 2) were obtained from the transmission IR spectra for colloidal silica suspensions sandwiched between two $\mathrm{CaF}_{2}$ plates by the above calculation procedures. A broad absorption band around $3400 \mathrm{~cm}^{-1}$ and a small band at $1640 \mathrm{~cm}^{-1}$ are assigned to $\mathrm{OH}$ stretching and $\mathrm{H}-\mathrm{O}-\mathrm{H}$ bending vibrations of water molecules, respectively [41]. A sharp band at $1120 \mathrm{~cm}^{-1}$ can be assigned to $\mathrm{Si}-\mathrm{O}$ stretching vibration of colloidal silica particles [42]. A small band at $2350 \mathrm{~cm}^{-1}$ is due to atmospheric $\mathrm{CO}_{2}$.

\section{B. Difference Absorption index $k$ spectra from pure bulk water}

In order to examine changes of $\mathrm{O}-\mathrm{H}$ stretching band shape, difference absorption index $k$ spectra from the pure water spectrum for each silica colloid volume fraction were calculated (Fig. 3). The $k$ spectra were first normalized to the peak height at $\mathrm{H}-\mathrm{O}-\mathrm{H}$ bending band $\left(1640 \mathrm{~cm}^{-1}\right)$ of colloidal silica suspensions in order to eliminate the bulk water and extract the spectroscopic feature of the interfacial water. The bending band has been often used as an indicator of bulk water because of its less sensitivity for the hydrogen bond structure than the stretching band [43].

These difference $k$ spectra show the following changes with increasing silica colloid volume fraction (Fig. 3):

1. Decrease in the intensity around $3380 \mathrm{~cm}^{-1}$

2. Increase in the intensity around $3620 \mathrm{~cm}^{-1}$

3. Increase in the intensity around $3060 \mathrm{~cm}^{-1}$ 


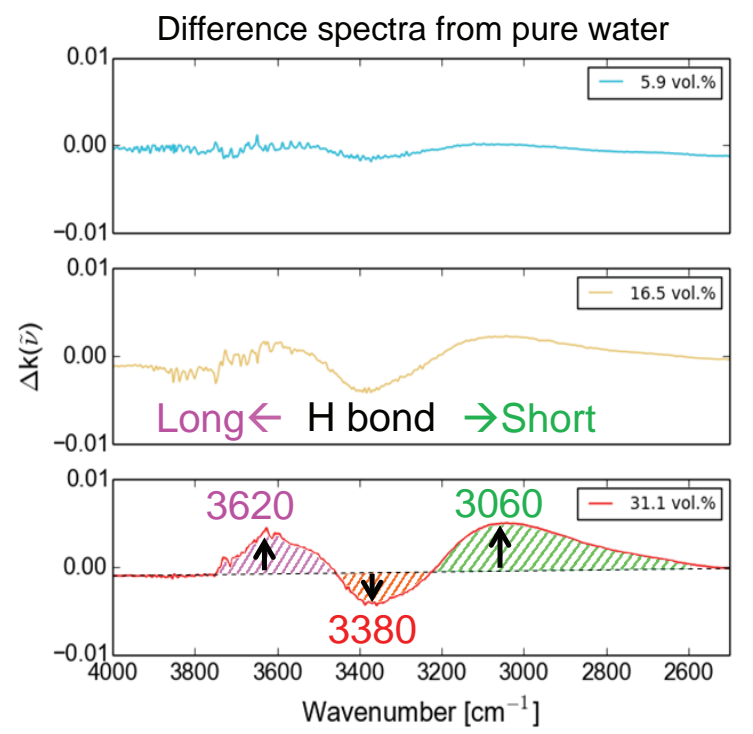

FIG. 3. Difference absorption index $k$ spectra for colloidal silica suspensions with three representative volume fractions by the following subtraction method of pure water. The pure water spectra were subtracted from the $k$ spectra of colloidal silica so as to set the peak height at $1640 \mathrm{~cm}^{-1}(\mathrm{H}-\mathrm{O}-\mathrm{H}$ bending) zero. The bands around 3620 and $3060 \mathrm{~cm}^{-1}$ increase and that around $3380 \mathrm{~cm}^{-1}$ decreases with increasing silica volume fraction.

The decreasing intensity at around $3380 \mathrm{~cm}^{-1}$ in the difference $k$ spectra for increasing silica colloid volume fraction (Fig. 3) may correspond to a loss of the bulk water feature in the interfacial water. On the other hand, the increasing intensity at around 3620 and $3060 \mathrm{~cm}^{-1}$ for increasing silica colloid volume fraction can be attributed to characteristics of the surface and interfacial species. The 3620 and $3060 \mathrm{~cm}^{-1}$ components correspond to $\mathrm{OH}$ species with longer and shorter $\mathrm{H}$ bonds, respectively, based on the relation of $\mathrm{O}-\mathrm{H}$ band frequency and $\mathrm{H}$ bond distance $[10]$.

\section{3620,3060 and $3380 \mathrm{~cm}^{-1}$ band components}

The band areas of these interfacial water components at the different volume fractions of silica were calculated (Fig. 4). The band area of the $3380 \mathrm{~cm}^{-1}$ component $\left(3455-3220 \mathrm{~cm}^{-1}\right)$ decreases with the silica volume fraction. On the other hand, the band areas of the $3620 \mathrm{~cm}^{-1}$ (4000-3455 $\left.\mathrm{cm}^{-1}\right)$ and the $3060 \mathrm{~cm}^{-1}\left(3220-2500 \mathrm{~cm}^{-1}\right)$ components increase with the silica volume fraction.

The increase of the $3060 \mathrm{~cm}^{-1}$ component appears to be proportional to the silica volume fraction. However, the increase of the $3620 \mathrm{~cm}^{-1}$ component and the decrease of the $3380 \mathrm{~cm}^{-1}$ component show less linearities. Although data are somewhat scattered, these two components might show some saturations at higher silica volume fractions.

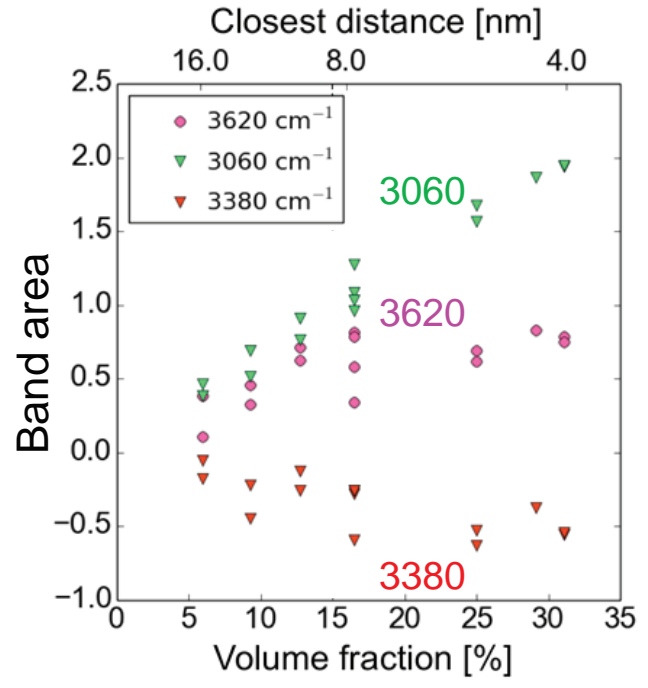

FIG. 4. Band areas of residual components in the difference $k$ spectra for colloidal silica suspensions in Fig. 3 (shaded area). The closest distance between silica particles is calculated by assuming cubic closest packing (сcp) and indicated at the upper horizontal axis.

\section{DISCUSSION}

\section{A. Interfacial and surface $\mathrm{OH}$ components}

The obtained results showed increase/decrease of three $\mathrm{OH}$ components at 3620,3380 and $3060 \mathrm{~cm}^{-1}$ in the difference spectra from bulk water with increasing silica volume fractions (Fig. 4). Since the $3380 \mathrm{~cm}^{-1}$ component decrease with silica volume fraction, this component can correspond to the bulk water. In fact, $\mathrm{OH}$ band for pure water has a band maximum around $3400 \mathrm{~cm}^{-1}$ (Fig. 2). This bulk component is corresponding to "liquid-like" water component around $3400 \mathrm{~cm}^{-1}$ with random orientations observed in SFG measurements of water at silica/water interfaces $[15,16]$.

The $3620 \mathrm{~cm}^{-1}$ component is considered to be longer $\mathrm{H}$ bond water molecules than the bulk "liquid-like" water. The band position is somewhat lower than the $3690 \mathrm{~cm}^{-1}$ component observed by SFG, which is called "free $\mathrm{OH} "$ [15].

The $3060 \mathrm{~cm}^{-1}$ component corresponds to shorter $\mathrm{H}$ bond water molecules than the bulk. This component was not clearly observed in SFG spectra but can be obscured by the intense band around $3200 \mathrm{~cm}^{-1}$ due to "icelike" water at the silica surface $[15,16]$. The absence of $3200 \mathrm{~cm}^{-1}$ component in our transmission spectra might be due to the general presence of this "ice-like" component throughout the silica/water system. On the other hand, oriented "ice-like" water molecules on the silica surface were selectively observed in SFG spectra.

Sulpizi et al. (2012) calculated IR spectra for interfacial water by density functional theory molecular dynamics simulations (DFTMD) [29]. Two bands around 3080 and $3200 \mathrm{~cm}^{-1}$ were found for surface silanol groups, which are strongly hydrogen bonded each other. They reported also that water molecules bonded to out-of plane and in-plane surface silanols are located at $3160 \mathrm{~cm}^{-1}$ (ice-like) and 
$3300 \mathrm{~cm}^{-1}$ (liquid-like), respectively. Subsequent water layers show absorption bands around $3280 \mathrm{~cm}^{-1}$ with a shoulder around $3550 \mathrm{~cm}^{-1}[29]$.

Therefore, the $3060 \mathrm{~cm}^{-1}$ component observed in our study can correspond to surface silanol component around $3080 \mathrm{~cm}^{-1}$ reported by Sulpizi et al. (2012) [29]. On the other hand, the $3620 \mathrm{~cm}^{-1}$ component might correspond to weakly hydrogen bonded subsequent water layers after the first water layers strongly hydrogen bonded to the surface silanols.

If the $3060 \mathrm{~cm}^{-1}$ component is due to in-plane surface silanol which is strongly $\mathrm{H}$ bonded to adjacent out-of plane surface silanol, in-plane $\mathrm{Si}-\mathrm{OH}$ vibrations could not be observed by SFG, because of its selectivity to the $\mathrm{OH}$ dipole moments normal to the surface plane.

\section{B. Evaluation of closest distance among silica particles}

In order to discuss origins of increase/decrease of the 3620,3380 and $3060 \mathrm{~cm}^{-1}$ band areas with increasing silica volume fraction, the closest distance among silica particles was evaluated by assuming the closest cubic packing (cсp). The closest distance among silica particles is indicated in the upper part of the horizontal axis of Fig. 4. The distance decreases from $16 \mathrm{~nm}$ at 5 vol\% to $4 \mathrm{~nm}$ at 31 vol.\% silica (Fig. 4). The possible saturation of the $3620 \mathrm{~cm}^{-1}$ component over 15 vol.\% of silica colloids corresponds to the closest distance of less than about $10 \mathrm{~nm}$.

\section{Electrical double layer model}

If the 3620 and $3380 \mathrm{~cm}^{-1}$ components do not continue to increase/decrease linearly with the silica volume fraction, these might be related to overlapping electrical double layer (EDL) developed around colloidal silica particles. The spreading length of the diffuse layer, the outer layer of EDL, can be characterized by the Debye length [24]. Since the initial colloidal silica suspension (16.5 vol.\%) includes $\mathrm{Na}_{2} \mathrm{SO}_{4}$ as a stabilization agent, the Debye length equation for a salt with cation : anion molar ratio of $2: 1$ is:

$$
\kappa^{-1}=1.76 \times 10^{-10}\left(C_{\text {salt }}\right)^{-\frac{1}{2}}[\mathrm{~m}]
$$

The concentration of $\mathrm{Na}_{2} \mathrm{SO}_{4} C_{\text {salt }}[\mathrm{mol} / \mathrm{L}]$ can be calculated for each volume fraction of the colloidal silica solutions based on the initial concentration at 16.5 vol. $\%$ ( 0.02 wt.\%). The obtained Debye length is plotted against the silica volume fraction in Fig. 5.

Since the diffuse layer spreads from each silica particle surface, the closest distance of silica particles (Fig. 4) should be divided by 2 to compare with the Debye length. The half closest distance among silica colloid particles is plotted in Fig. 5. The Debye length (red curve) crosses the half closet distance (blue curve) at $5 \mathrm{~nm}$ for about 13 vol.\% of silica. Therefore, electrical double layers around silica particles can start overlapping from about $13 \mathrm{vol} \%$ with the closet distance of about $5 \mathrm{~nm}$. This overlap might explain the attenuation of increase/decrease of 3060 and $3380 \mathrm{~cm}^{-1}$ components at larger silica volume fractions (Fig. 5).

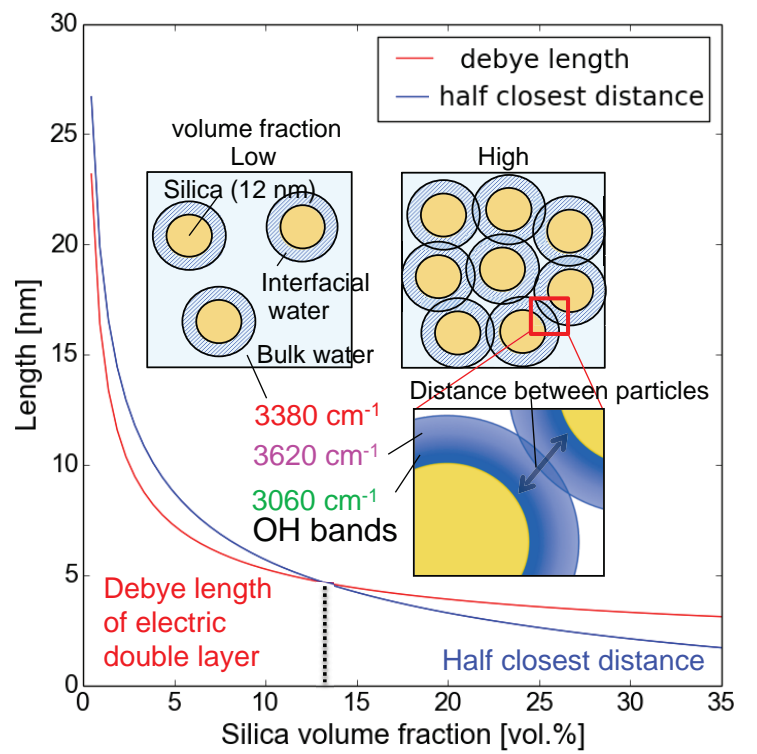

FIG. 5. The half of the closest distance among silica particles (blue curve) and the Debye length of the electrical double layer (red curve) as functions of the silica volume fraction. Schematic images of interfacial water surrounding silica particles are also indicated. Electrical double layers around silica particles become overlapped with increasing silica volume fraction over 13 vol.\% by decreasing the half closest distance less than about $5 \mathrm{~nm}$ (total distance of $10 \mathrm{~nm}$ ). The 3380,3620 and $3060 \mathrm{~cm}^{-1}$ bands can correspond to bulk, outer interfacial and inner interfacial $\mathrm{OH}$ species, respectively.

The different behavior of $3620 \mathrm{~cm}^{-1}$ and $3060 \mathrm{~cm}^{-1}$ components with increasing colloidal silica concentrations observed in this study (Fig. 4) can be explained by the following model: The lower wavenumber component $\left(3060 \mathrm{~cm}^{-1}\right)$ are confined near the silica surface and can increase with increasing colloidal silica concentrations (Fig. 5). This can correspond to surface silanol component around $3080 \mathrm{~cm}^{-1}$ reported by Sulpizi et al. (2012) [29]. On the other hand, the higher wavenumber water component $\left(3620 \mathrm{~cm}^{-1}\right)$ would extend into the diffuse layer over the Debye length and would be saturated by the overlapping of diffuse layer among silica particles (Fig. 5). Therefore, this $3620 \mathrm{~cm}^{-1}$ component might correspond to weakly hydrogen bonded subsequent water layers after the first water layers strongly hydrogen bonded to the surface silanols.

In the overlapping electrical double layers, high concentrations of $\mathrm{H}^{+}$and $\mathrm{Na}^{+}$cations can be attained and these can be adsorbed on the negatively charged silica surface at slightly alkaline environment of the present study $(\mathrm{pH}=8.2)$. Water molecular structures might be then modified according to the surface species of silica [15].

The confinement of water molecules in small nanometer-scale pore spaces may cause structural modification of water associated with the electrical double layer developing on the material surfaces. These models can be discussed more precisely and quantitatively by more experimental data by controlling the electrical double layers and surface species together with the simulation such as molecular dynamics (MD) calculation. 


\section{CONCLUSIONS}

The difference absorption index $k$ spectra from the pure water were extracted from transmission infrared spectra on silica colloid suspensions with varying volume fractions (0-31.1 vol.\%) sandwiched between two $\mathrm{CaF}_{2}$ plates of $1 \mathrm{~mm}$ thick under an infrared microscope. The difference spectra in the $\mathrm{O}-\mathrm{H}$ stretching region have three components showing different behavior with increasing silica volume fraction: the $3060 \mathrm{~cm}^{-1}$ component increased linearly with the silica volume fraction, while the $3620 \mathrm{~cm}^{-1}$ and $3380 \mathrm{~cm}^{-1}$ components increased/decreased with less linearities. The attenuation of these increase/decrease appears to correspond to the half closest distance of about $5 \mathrm{~nm}$ indicating overlapping electrical double layers. The $3060 \mathrm{~cm}^{-1}$ and $3620 \mathrm{~cm}^{-1}$ components are considered to be characteristic to the surface silanol and interfacial water and affected by overlapping electrical double layers among silica particles.
[1] G. E. Brown, Science 294, 67 (2001).

[2] N. Nandi, K. Bhattacharyya, and B. Bgchi, Chem. Rev. 100, 2013 (2000).

[3] D. O. De Haan, T. Brauers, K. Oum, J. Stutz, T. Nordmeyer, and B. J. Finlayson-Pitts, Int. Rev. Phys. Chem. 18, 343 (1999).

[4] A. W. Adamson, Physical Chemistry of Surfaces, 2nd ed. (Interscience Publishers Inc., New York, 1990).

[5] J. N. Isaraelachvili, Intermolecular and surface forces, Third Edition (Academic Press, London, 2011).

[6] H. Sakuma, K. Otsuki, and K. Kurihara, Phys. Rev. Lett. 96, 046104 (2006).

[7] M. F. Toney, J. N. Howard, J. Richer, G. L. Borges, J. G. Gordon, O. R. Melroy, D. G. Wesier, D. Yee, and L. B. Sorensen, Nature 368, 444 (1994).

[8] A. A. Skelton, P. Fenter, J. D. Kubicki, D.J. Wesolowski, and P. T. Cummings, J. Phys. Chem. C 115, 2076 (2011).

[9] Y. Wang, L. Wang, M. A. Hampton, and A. V. Nguyen, J. Phys. Chem. 117, 2113 (2013).

[10] K. Nakamoto, M. Margoshes, and R. E. Rundle, J. Am. Chem. Soc. 77, 6480 (1955).

[11] Q. Du, R. Superfine, E. Freysz, and Y. R. Shen, Phys. Rev. Lett. 70, 2313 (1993).

[12] Q. Du, E. Freysz, and Y. R. Shen, Phys. Rev. Lett. 72, 238 (1994).

[13] Q. Du, E. Freysz, and Y. R. Shen, Science 264, 826 (1994).

[14] Y. R. Shen, Solid State Commun. 108, 399 (1998).

[15] S. Ye, S. Nihonyanagi, and K. Uosaki, Phys. Chem. Chem. Phys. 3, 3463 (2001).

[16] V. Ostroverkhov, G. A. Waychunas, and Y. R. Shen, Chem. Phys. Lett. 386, 144 (2004).

[17] M. Sovago, R. K. Campen, H. J. Bakker, and M. Bonn, Chem. Phys. Lett. 470, 7 (2009).

[18] S. Nihonyanagi, S. Yamaguchi, and T. Tahara, J. Am. Chem. Soc. 132, 6867 (2010).

[19] S. Nihonyanagi, T. Ishiyama, T-K. Lee, S. Yamaguchi, M. Bonn, A. Morita, and T. Tahara, J. Am. Chem. Soc. 133, 16875 (2011).

[20] D. Argyris, D. R. Cole, and A. Strilolo, ACS Nano 4, 2035 (2010).

[21] Y. Leng, Langmuir 28, 5339 (2012).
[22] I. C. Bourg and C. I. Steefel, J. Phys. Chem. C 116, 11556 (2012).

[23] H. Zhu, A. Ghoufi, A. Szymczyk, B. Balannec, and D. Morineau, Phys. Rev. Lett. 109, 107801 (2012).

[24] R. B. Schoch, J. Han, and P. Renaud, Rev. Mod. Phys. 80, 839 (2008).

[25] J. R. Bailey and M. M. McGuire, Langmuir 23, 10995 (2007).

[26] S. Le Caër, S. Pin, S. Esnouf, Q. Ph. Raffy, J. Renault, J.B. Brubach, G. Creff, and P. Roy, J. Phys. Chem. Chem. Phys. 13, 17658 (2011).

[27] K. Yamashita and H. Daiguji, J. Phys. Chem. C 117, 2084 (2013).

[28] J. Yang and E. G. Wang, Phys. Rev. B 73, 035406 (2006).

[29] M. Sulpizi, M. Gaigeot, and M. Sprik, J. Chem. Theory Comput. 8, 1037 (2012).

[30] N. Nishiyama and T. Yokoyama, Procedia Earth and Planetary Science 7, 620 (2013).

[31] S. Ochiai, in: Introduction to Experimental Infrared Spectroscopy, Eds. M. Tasumi and A. Sakamoto, (Wiley, Chichester, 2015), p. 179.

[32] J. E. Bertie and Z. Lan, Appl. Spectrosc. 50, 1047 (1996).

[33] C. D. Keefe, T. Wilcox, and E. Campbell, J. Mol. Struct. 1009, 111 (2012).

[34] M. H. A. Kramers, Atti Cong. Intern. Fisici. 2, 545 (1927).

[35] R. de L. Kronig, J. Opt. Soc. Am. Rev. Sci. 12, 547 $(1926$,$) .$

[36] K. Alexander, A. Killey, G. Meeten, and M. Senior, J. Chem. Soc. Faraday Trans. 2 77, 361 (1981).

[37] M. Daimon and A. Masumura, Appl. Opt. 46, 3811 (2007).

[38] I. H. Malitson, J. Opt. Soc. Am. 55, 1205 (1965).

[39] J. P. Hawranek, P. Neelakantan, R. P. Young, and R. N. Jones, Spectrochimica Acta A 32, 75 (1976).

[40] I. H. Malitson, Appl. Opt. 2, 1103 (1963).

[41] S. Y. Venyaminov and F. G. Prendergast, Anal. Biochem. 248, 234 (1997).

[42] H. A. Benesi and A. C. Jones, J. Phys. Chem. 63, 179 (1959).

[43] D. V. Asay and S. H. Kim, J. Phys. Chem. B 109, 16760 (2005). 